The University of San Francisco

USF Scholarship: a digital repository@ Gleeson Library |

Geschke Center

Mathematics

College of Arts and Sciences

2009

\title{
Evolution of Cooperation through the Heterogeneity of Random Networks
}

Stephen Devlin

University of San Francisco, smdevlin@usfca.edu

T Treloar

Follow this and additional works at: http://repository.usfca.edu/math

Part of the Mathematics Commons

\section{Recommended Citation}

Devlin, S., \& Treloar, T. (2009). Evolution of cooperation through the heterogeneity of random networks. Phys. Rev. E 79, 16-107. http://dx.doi.org/10.1103/PhysRevE.79.016107

This Article is brought to you for free and open access by the College of Arts and Sciences at USF Scholarship: a digital repository @ Gleeson Library | Geschke Center. It has been accepted for inclusion in Mathematics by an authorized administrator of USF Scholarship: a digital repository@ Gleeson Library $\mid$ Geschke Center. For more information, please contact repository@usfca.edu. 


\title{
Evolution of cooperation through the heterogeneity of random networks
}

\author{
Stephen Devlin ${ }^{1}$ and Thomas Treloar ${ }^{2}$ \\ ${ }^{1}$ Mathematics Department, University of San Francisco2130 Fulton Street, San Francisco, California 94117, USA \\ ${ }^{2}$ Mathematics Department, Hillsdale College33 East College Street, Hillsdale, Michigan 49242, USA
}

(Received 5 August 2008; published 21 January 2009)

\begin{abstract}
We use the standardized variance $\left(\nu_{s t}\right)$ of the degree distribution of a random network as an analytic measure of its heterogeneity. We show that $\nu_{s t}$ accurately predicts, quantitatively, the success of cooperators in an evolutionary prisoner's dilemma. Moreover, we show how the generating functional expression for $\nu_{s t}$ suggests an intrinsic interpretation for the heterogeneity of the network that helps explain local mechanisms through which cooperators thrive in heterogeneous populations. Finally, we give a simple relationship between $\nu_{s t}$, the cooperation level, and the epidemic threshold of a random network that reveals an appealing connection between epidemic disease models and the evolutionary prisoner's dilemma.
\end{abstract}

DOI: 10.1103/PhysRevE.79.016107

PACS number(s): 89.75.Hc, 87.23.Kg, 02.50.Le, 89.75.Fb

\section{INTRODUCTION}

The versatility of the prisoner's dilemma (PD) as a relevant paradigm for interdisciplinary problems involving the study of conflict and cooperation has led to a vast body of research [1-6]. Indeed, the PD has become a standard tool in biology, economics, the social sciences, physics, and mathematics in which to study cooperation phenomena [1-7]. A great deal of recent attention has focused on a deepening understanding, via the evolutionary PD, of how and why cooperative behavior can emerge, thrive, and even dominate in certain frameworks despite a clear temptation for individuals to maximize their own fitness by acting selfishly $[2-5,7]$.

In its simplest form, the PD pits two agents against each other. Agents simultaneously choose between two strategies: $C$ for cooperate and $D$ for defect. The essence of the game is captured in the payoffs associated with the four possible states. Setting $b>1>0 \geqslant a$, PD payoffs are distributed according to the normalized symmetric matrix (where payoffs go to the row-player):

\begin{tabular}{|l|l|l|}
\hline & $C$ & $D$ \\
\hline$C$ & 1 & $a$ \\
\hline$D$ & $b$ & 0 \\
\hline
\end{tabular}

It is clear from the payoff inequalities that a rational (selfinterested) agent choosing independently has no incentive to play $C$. Indeed, the Nash equilibrium predicts that both agents will defect. The dilemma arises from the inefficiency of the equilibrium: both players could benefit (i.e., enjoy higher payoffs than at equilibrium) via mutual cooperation [3].

The PD has been further studied in the setting of evolutionary replicator dynamics. In this context, a strategy spreads in a population according to its fitness and the repeated interactions between agents are assumed to be random and well mixed - any agent is equally likely to interact with any other agent in the population. In such a setting, cooperation vanishes over time and the (evolutionarily stable) equilibrium rests with all agents defecting [3].
Abandoning the well-mixed scenario led to important advances. In the seminal work of Nowak and May [8], agents playing an evolutionary PD were placed at vertices in a lattice, with interactions limited to neighbors (vertices connected by edges). It was found that a nontrivial population of cooperators could become evolutionarily stable over some game parameter values.

This work led to the recognition that agent-agent interactions could be better modeled by introducing topological constraints on a population in the form of a graph, with vertices representing agents and edges indicating a relationship along which an interaction can take place. The subsequent result that spatial structure could drive cooperation in the evolutionary PD sparked intense study of the effects of varied network topologies on cooperation phenomena.

More recently [9-17], considerable attention has been given to the (discrete replicator) dynamics of an evolutionary PD on a network. Of particular interest is the now wellestablished result that so-called scale-free distributions (where the degrees of vertices follow a power-law distribution) greatly benefit cooperation in the PD, as well as variants like the snowdrift game. In fact, cooperation can dominate on the network over all parameter values of the game on certain networks such as the Barbási-Albert scale-free network generated via growth and preferential attachment [10].

The distinguishing characteristic of power-law distributions is that they are "heterogeneous;" the connectivity of individual agents varies widely across the network [18-20]. Many factors ultimately influence the complex dynamics of an evolutionary game on such networks, but it is wellestablished that heterogeneity in particular enhances the ability of cooperators to survive and thrive. While progress has been made in understanding the particulars of these complex dynamics [9], many questions remain.

This paper studies a quantifiable measure of heterogeneity that accords well with cooperation phenomena in the sense that more heterogeneity corresponds to more cooperation in an evolutionary PD in an explicit and predictable way. Also, a further explanation as to the mechanisms through which heterogeneity fosters enhanced cooperation is given. A primary focus, therefore, is on the influence of the degree distribution of a network on cooperation phenomena, which is 
addressed using generating functions associated to random graphs.

A simple, quantifiable measure of heterogeneity on a network with no degree-degree correlations is the standardized variance $\left(\nu_{s t}\right)$. This value is easily computed from the degree distribution and can be compared across networks with varying average degree: a particularly nice property not shared by some alternative measures of heterogeneity [15]. Monte Carlo simulations are used to confirm that $\nu_{s t}$ is an excellent predictor of average cooperation (appropriately weighted) in the evolutionary PD on the network.

Moreover, standardized variance can be interpreted, using generating functions on random graphs, in terms of the relationship between the average number of interactions of the agents in the population and the average number of interactions of neighboring agents in the population. This interpretation gives an intrinsic explanation of those properties that are critical for enhanced cooperation in a network.

Finally, $\nu_{s t}$ is shown to be closely related to the network's epidemic threshold. A simple and intuitive functional relationship between average network cooperation in the PD and the epidemic threshold of the network is given, relating two important frameworks in the study of complex systems.

\section{PRELIMINARIES}

A network, $\mathcal{N}$, is an undirected graph consisting of vertices and edges connecting vertices. A network is not permitted loops (edges connecting a vertex to itself), nor multiple edges (more than one edge connecting any pair of vertices). The degree of a vertex is defined to be the number of edges that emanate from that vertex.

Let $X$ be the random variable that assigns to a vertex in the network the degree of that vertex. Let $p_{k}$ denote the probability that $X$ takes the value $k$, i.e., $p_{k}$ is the probability that a random vertex has degree $k$, with $k$ in the set of all possible vertex degrees. The probability generating function for $X$ (i.e., for the degrees of randomly chosen vertices) is defined to be

$$
G(x)=\sum_{k>0} p_{k} x^{k}
$$

Averaging over the probability distribution gives the average degree of vertices in a network:

$$
G^{\prime}(1)=\sum_{k>0} k p_{k}=\langle k\rangle .
$$

The correspondence between graphs and degree generating functions is many-to-one: distinct graphs can and do share the same generating function. In this paper, we consider networks with a fixed generating function, but that are random in all other respects. That is, given a fixed degree distribution, the collection of all graphs that share this distribution is considered and networks chosen uniformly at random from this collection are studied. Consequently, results gleaned from generating functions represent an average over the collection of all random networks sharing that fixed degree distribution [21]. This assumption implies that the networks considered are completely determined by their degree distribution. There are no correlations between the degrees of vertices sharing an edge: the probability that a degree $j$ vertex is connected to a degree $k$ vertex is independent of $k$ and the correlation coefficient of the degrees of vertices at the ends of edges is zero. It is worth noting that while this independence assumption gives a degree-degree correlation coefficient of zero, it is not the case that a correlation coefficient of zero implies independence.

When a random edge is chosen from the network, it is more likely to lead to a higher degree vertex. The probability that a random edge leads to a vertex of degree $k$ is proportional to $k$. Therefore, if $Y$ is the random variable taking values in the set of possible degrees of vertices reached along edges, then the generating function for $Y$ is given by

$$
T(x)=\frac{\sum_{k>0} k p_{k} x^{k}}{\sum_{k>0} k p_{k}}=\frac{x G^{\prime}(x)}{G^{\prime}(1)} .
$$

When a vertex is chosen at random, and then a randomly chosen edge emanating from that vertex is followed, a neighbor is reached. Making use of the independence assumption for degree-degree correlations, it follows that the generating function for the degree of randomly chosen neighbors is also given by $T(x)$, and the average degree of these neighbors is

$$
T^{\prime}(1)=\frac{\sum_{k>0} k^{2} p_{k}}{\sum_{k>0} k p_{k}}=\frac{\left\langle k^{2}\right\rangle}{\langle k\rangle} .
$$

It is important to note that the average degree of neighbors is always greater than or equal to the average degree of the vertices in the network, with equality only when all vertices on the network have the same degree. From now on $T^{\prime}(1)$ is referred to as the average degree of neighbors in the network. In the following, the configuration model algorithm of [28] is used to generate random uncorrelated networks consistent with a fixed degree distribution.

\section{NETWORKS AND HETEROGENEITY}

Let $\mathcal{N}$ be a network with degree generating function $G(x)$ associated to the degree random variable $X$. Consider the variance of $X$ :

$$
\operatorname{Var}[X]=\left\langle k^{2}\right\rangle-\langle k\rangle^{2},
$$

where $\left\langle k^{2}\right\rangle$ is the expected value of $X^{2}$ and $\langle k\rangle$ is the expected value of $X$. It follows that

$$
\sum_{k} k p_{k}=\langle k\rangle=G^{\prime}(1)
$$

and

$$
\sum_{k} k^{2} p_{k}=\left\langle k^{2}\right\rangle=G^{\prime}(1) T^{\prime}(1)
$$

Therefore

$$
\operatorname{Var}[X]=G^{\prime}(1) T^{\prime}(1)-G^{\prime}(1)^{2}=G^{\prime}(1)\left[T^{\prime}(1)-G^{\prime}(1)\right] .
$$

The variance of the degree distribution is the average degree of a randomly chosen vertex multiplied by the difference between the average degree of a randomly chosen neighbor 
and the average degree of a randomly chosen vertex.

In order to arrive at a unitless numerical measure of heterogeneity, variance is normalized by dividing by

$$
\langle k\rangle^{2}=G^{\prime}(1)^{2} .
$$

This gives the standardized variance $\left(\nu_{s t}\right)$ of $X$ :

$$
\nu_{s t}=\frac{\left\langle k^{2}\right\rangle-\langle k\rangle^{2}}{\langle k\rangle^{2}}=\frac{T^{\prime}(1)-G^{\prime}(1)}{G^{\prime}(1)} .
$$

Standardized variance will be used as the numerical measure of the heterogeneity of the network. As a result, heterogeneity has the interpretation of giving the difference between the average size of a neighbor and the average size of a vertex in relation to the average size of a vertex. In this way, $\nu_{s t}$ is like a $Z$ score, giving the number of "average degrees" between a random vertex and his neighbor. Standardized variance has been recognized as a measure of network heterogeneity in the context of network effects on epidemiology [22], a connection that will be discussed later.

In order to explore the relationship between $\nu_{s t}$ and cooperation in an evolutionary PD, networks with varying degrees of heterogeneity are required. To this end, two families of networks are considered.

The first family is generated using the algorithm in [24] that interpolates between the Barbási-Albert model $[18,19]$ and Erdős-Rényi random graphs [25-27]. The BarbásiAlbert (BA) model gives rise to heterogeneous networks, while Erdős-Rényi (ER) random graphs are far more homogeneous. Each member of this family of networks will have $M$ vertices, average degree $2 m$, and will be determined by a single scalable parameter $\alpha$ between 0 and 1 .

The BA-ER family of graphs is constructed starting from a complete graph on $n_{0}$ vertices. A new vertex is chosen from the remaining set of all $M-n_{0}$ unconnected vertices. The new vertex has $m$ edges to attach in the following way: with probability $\alpha$, the vertex connects to any of the $M-1$ network vertices with a uniform probability and, with probability $1-\alpha$, the edge attaches to an existing network vertex with probability proportional to the current degree of the existing vertex (i.e., by preferential attachment). The procedure is repeated $m$ times for a particular vertex, once for each edge.

When $\alpha=0$, this algorithm becomes the Barbási-Albert growth and preferential attachment algorithm of [18], and the resulting network has a degree distribution that follows a power law, $p_{k} \sim \frac{1}{k^{3}}$. When $\alpha=1$, an Erdős-Rényi (ER) random network with Poisson degree distribution is obtained. For 0 $<\alpha<1$, the graph is a hybrid of the two with intermediate heterogeneity.

Networks generated in this way (with $\alpha<1$ ), however, are not random in that the degree of a random vertex and a random neighbor are not independent. Therefore, after a network is generated, it is distilled down to its degree distribution by throwing away all specific contact information. The configuration model algorithm [28] is used to reconfigure a random network consistent with the specified degree distribution. In the configuration model, vertices are generated with stubs emanating from them which will become the ends of edges. The number of stubs is determined by the degree

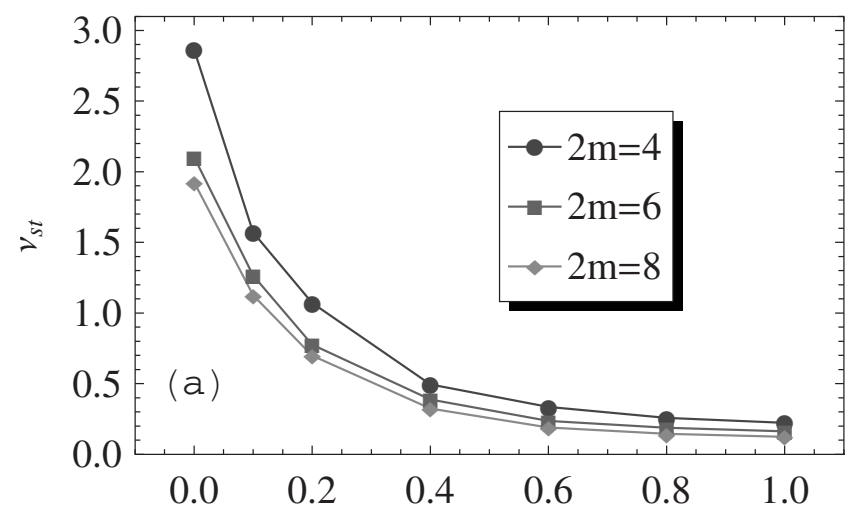

$\alpha$

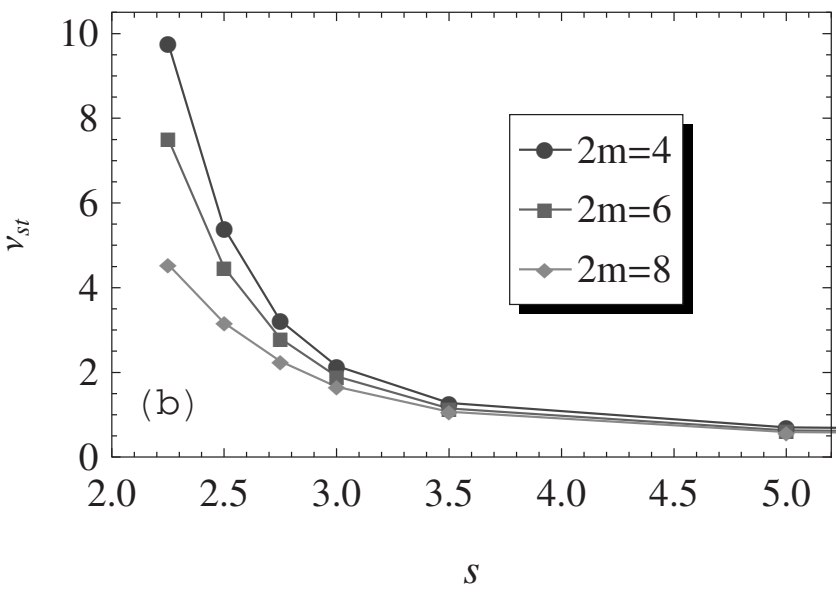

FIG. 1. Standardized variation, $\nu_{s t}$, as a function of the parameter $\alpha$ for the $B_{2 m, \alpha}$ family of networks (a), and as a function of the tail parameter $s$ for the $Z_{2 m, s}$ family (b).

distribution. Edges are then formed by randomly connecting stubs to form complete edges until all stubs are used, resulting in a random network with the given degree distribution.

Via this algorithm, networks with $10^{4}$ vertices are generated that are distinguished by two parameters: $2 \mathrm{~m}$ (average degree) and $\alpha$, with $2 m \in\{4,6,8\}$ and $\alpha \in\{0.0,0.10,0.20,0.40,0.60,0.80,1.00\}$. Finally, for each pair of parameter choices, three distinct graphs are generated and labeled $a, b$, and $c$, respectively, from three independent instances of the algorithm, followed by a configuration model on the resulting degree distributions.

This gives a total of 63 different graphs identified by three parameters. The graphs are denoted: $B_{2 m, \alpha, l}$ with

$$
2 m \in\{4,6,8\},
$$

$$
\alpha \in\{0.00,0.10,0.20,0.40,0.60,0.80,1.00\},
$$

and

$$
l \in\{a, b, c\} .
$$

Figure 1(a) shows the standardized variance of $B_{2 m, \alpha, l}$ as a function of $\alpha$ for each average degree, $2 m \in\{4,6,8\}$, averaged over the three networks, $l \in\{a, b, c\}$. (The actual analytical value of $\nu_{s t}$ for each individual network appears be- 
low.) Figure 1 illustrates the expected result that standardized variance, and thus heterogeneity, decreases as $\alpha$ increases. Consider the interpretation of standardized variance, $\nu_{s t}$, from Eq. (3) given in terms of the average degree of randomly chosen neighbors and the average degree of randomly chosen vertices in the network. For a fixed average vertex degree, as $\alpha$ decreases, the average size of neighbors increases. In the following section, this increase in the size of the average neighbor with respect to the size of the average degree will be discussed in detail in terms of its effects on cooperation phenomena on the network.

The second family of networks is generated with degree distributions that follow power laws that can be adjusted to allow for varied tail exponents, including those below the tail 3 seen in networks generated from the BA algorithm. Let $N$ denote the maximum possible vertex degree allowed in the network and let $2 m$ be the desired average degree. A degree sequence is generated using the Zipf-Mandelbrot distribution. The Zipf-Mandelbrot distribution has probability mass function:

$$
f(k, N, q, s)=\frac{1}{H_{N, q, s}(k+q)^{s}},
$$

where $H_{N, q, s}=\sum_{i=m}^{N} \frac{1}{(i+q)^{s}}$. The exponent $s$ gives the power law for the distribution and $q$ is chosen to ensure that the network has the correct average degree.

As above, networks with $10^{4}$ vertices are generated with average degree $2 m \in\{4,6,8\}$. For each $2 m$, we consider power laws determined by $s \in\{2.25,2.5,2.75,3,3.5,5,10\}$. The summation in Eq. (4) starts at $m$ to ensure that the minimum degree of a vertex is half the average degree in keeping with the BA-ER family of networks considered above. The maximum possible vertex degree is capped, for all networks in this family, by taking $N=600$. The degree sequence is generated from the cumulative distribution function $F$ associated to $f$ by determining, for each $k=1,2, \ldots, 10000$, the point in the domain of $F$ that maps to $\frac{k}{10001}$ in the image of $F$. The configuration model is again used to generate a random network with the specific degree distribution. For each choice of $2 m$ and $s$, three distinct graphs are generated for a total of 63 networks denoted $Z_{2 m, s, l}$, with $2 m$ and $s$ as above, and $l \in\{a, b, c\}$.

Figure 1(b) shows the standardized variance of $Z_{2 m, s, l}$ as a function of $s$ for each average degree $2 m \in\{4,6,8\}$, averaged over the three distinct graphs generated, given by $l \in\{a, b, c\}$. Note that the standardized variance, and thus heterogeneity, decreases as $s \geqslant 2.25$ increases. That is, heterogeneity decreases as the tail of the distribution becomes smaller. Referring to Eq. (3), as $s$ decreases to 2.25 and average degree is fixed, the average size of a neighbor increases. Actual $\nu_{s t}$ appears for all networks below.

\section{RESULTS AND DISCUSSION}

The standard version of the evolutionary prisoner's dilemma on a network $[9,10]$ is studied on the above networks with $10^{4}$ vertices. Following common practice, payoffs are normalized so that in the notation of the payoff matrix (1), $b=1+r$ and $a=0$. This means that the game depends on the single parameter $b=1+r$, and where the cost-to-benefit ratio $r$ runs between 0 and 1 in increments of 0.05 .

A round of play consists of all agents playing a pure strategy in a single PD game against all neighbors, with neighbor defined as any vertex with which the agent shares an edge. Agents collect and record their cumulative payoff each round and evolution is implemented according to the discrete ana$\log$ of the replicator dynamics [3,9]. An agent updates strategy by choosing a random neighbor for payoff comparison. If agent $v$ has degree $k_{v}$ and accumulated payoff $T_{v}$ and chooses neighbor $w$ having degree $k_{w}$ and payoff $T_{w}$, then $v$ adopts the strategy of $w$ with probability $P_{v \rightarrow w}$, where

$$
P_{v \rightarrow w}=\frac{\max \left\{0,\left(T_{w}-T_{v}\right)\right\}}{b \max \left\{k_{v}, k_{w}\right\}} .
$$

Thus an agent is more likely to be persuaded to change strategy when comparing with a more successful neighbor. Natural selection is reflected in the fact that fitter strategies are more likely to spread, and in the well-mixed population limit, simulation results converge to those of the traditional replicator dynamics.

Once each agent updates strategy, the process continues with another round of play and updating. Simulations proceed as follows. Starting from a random initial configuration, the system evolves for $10^{4}$ rounds of play, which is called a series. The average frequency of cooperators over the last 1000 rounds of a series is called the series mean. On each network, 100 series are run, each starting from a random initial configuration with cooperation probability of 0.5 . Finally, the equilibrium frequency of the cooperators is taken to be the average of the 100 series means.

Figures 2 and 3 show equilibrium levels of cooperators as a function of the game parameter $b=1+r$ for a sample of the graphs discussed above. These result are expected and agree with the well-established results seen in Refs. [10,14] on the BA-ER family of networks with degree correlations, as cooperation largely increases as $\alpha$ approaches zero within a fixed average degree.

For fixed average degree $2 m$, the $Z_{2 m, s, l}$ family also behaves in an intuitive fashion with respect to cooperation; the larger the tail (smaller $s$ ), the more "spread out" the degree distribution and, generally speaking, the more cooperation prevails on the network. In the following, we use the results of the previous sections to make explicit the dependence of cooperation on network heterogeneity as defined by $\nu_{s t}$.

In order to aggregate simulation results for a given network $\mathcal{N}$, a weighted average of cooperation levels on $\mathcal{N}$ is taken over all game parameter values. To reward networks that foster large scale cooperation despite strong temptation toward defection, the equilibrium cooperation level at game parameter $b=1+r$ is weighted by the cost-to-benefit ratio, $r$, of cooperation. The choice of $r$ as a weight is natural since one can choose game payoffs in a variety of qualitatively equivalent ways consistent with Eq. (1), all of which share the intrinsic parameter $r$. Moreover, since game payoffs in actual observed PD situations are notoriously difficult to pinpoint, an averaging process is appropriate. 

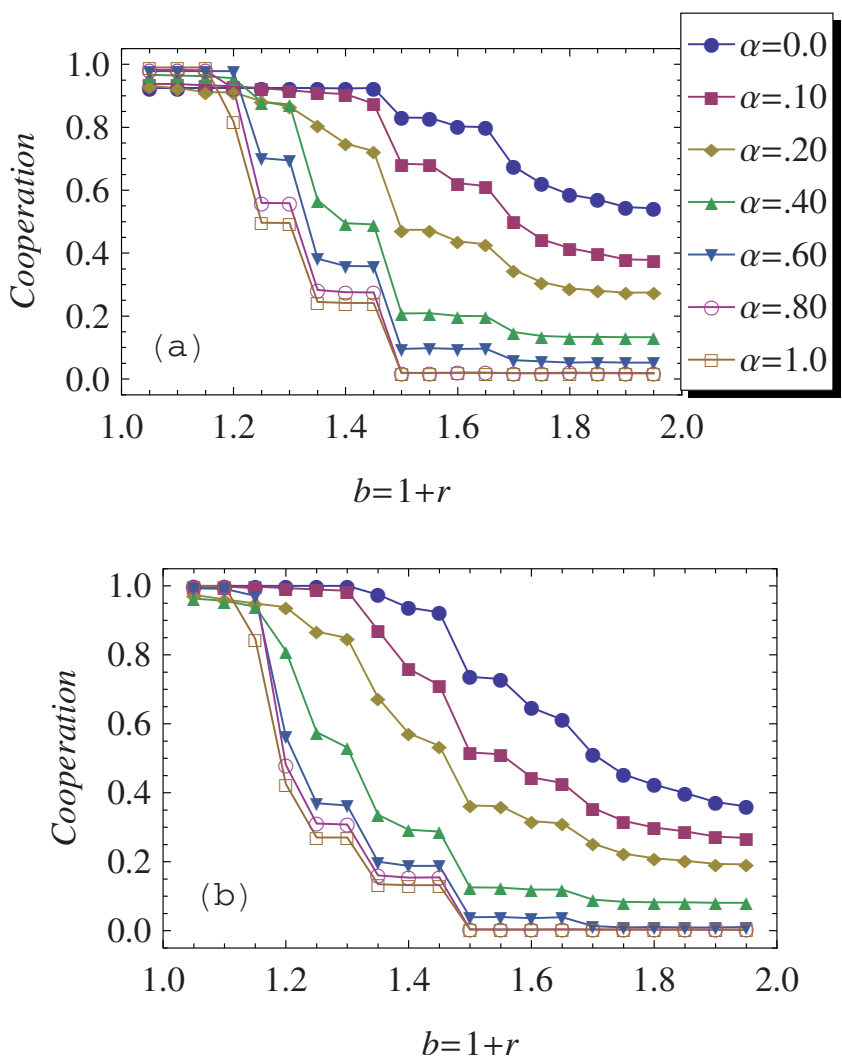

FIG. 2. (Color online) Equilibrium cooperation level as a function of the game parameter $b=1+r$ for the $B_{4, \alpha}$ (a) and $B_{6, \alpha}$ (b) families of networks.

For each network $\mathcal{N}$, let $c_{\mathcal{N}, r}$ denote the equilibrium cooperation level at PD parameter $b=1+r$. Let $\bar{c}_{\mathcal{N}}$ be the global average cooperation on the network over all $r$ values:

$$
\bar{c}_{\mathcal{N}}=\frac{\Sigma_{0<r<1} r c_{\mathcal{N}, r}}{\Sigma_{0<r<1} r} .
$$

The sums are taken over all cost-to-benefit ratios $r$ in increments of 0.05 . This gives a single quantitative measure of the global success of cooperators on the network, $\bar{c}_{\mathcal{N}}$, where the heterogeneity of $\mathcal{N}$ is given by $\nu_{s t, \mathcal{N}}$.

To each network $\mathcal{N}$ hosting an evolutionary PD is therefore associated an ordered pair consisting of heterogeneity of $\mathcal{N}$ and global average cooperation on $\mathcal{N}$ :

$$
\mathcal{N} \leftrightarrow\left(\nu_{s t, \mathcal{N}}, \bar{c}_{\mathcal{N}}\right) .
$$

Figure 4 shows a plot of these ordered pairs for each of the networks considered above [29]. The plot shows the existence of a strong positive functional relationship between standardized variation, $\nu_{s t}$, and average cooperation on a network. The precise relationship between $\nu_{s t}$ and average cooperation on a network is below.

In the literature, the effectiveness of heterogeneity as a conduit for cooperation on a network is often attributed to vertices of varying degree playing a disproportionate number of games each round, with particular attention given to the global role played by large vertices [9], also called hubs.
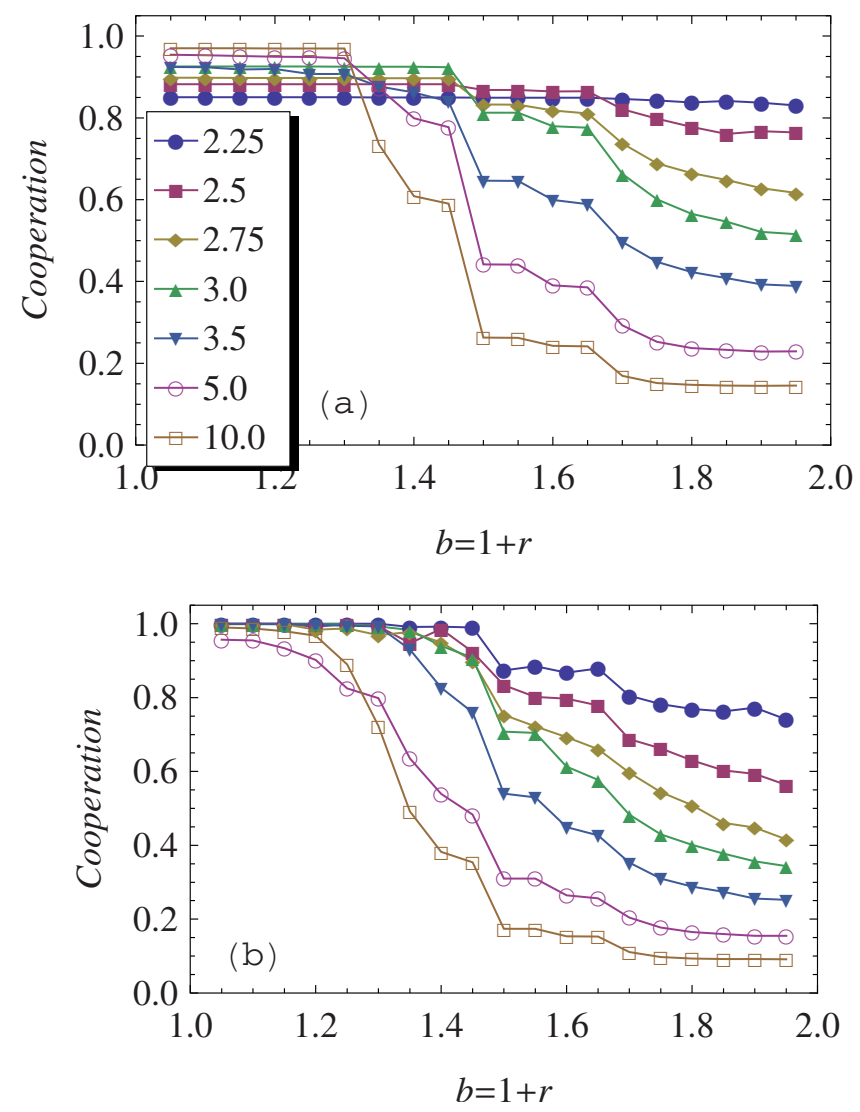

FIG. 3. (Color online) Equilibrium cooperation level as a function of the game parameter $b=1+r$ for the $Z_{4, s}$ family (a) and $Z_{6, s}$ family (b) of networks.

These hubs, by the nature of the PD and the updating scheme, eventually become influential cooperators who can spread cooperation to their many neighbors.

In addition, formula (3), derived by generating functions, suggests a more subtle local mechanism at work. By focusing attention on the difference between average degree of vertices and average degree of neighbors, one realizes that a strong cooperator is the only stable agent in the game. For example, while weak cooperators and weak defectors are both susceptible to strategy shifts, compare the position of a strong cooperator to a strong defector. In this context strong

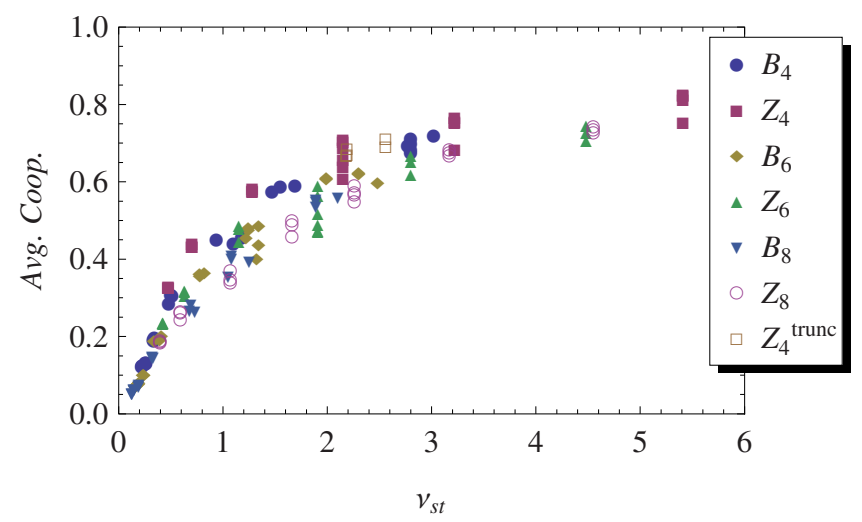

FIG. 4. (Color online) Average cooperation as a function of $\nu_{s t}$. 
means that the payoff of a vertex is relatively large given its total degree. By the nature of the payoff matrix, both agents must have a large number of cooperating neighbors relative to their total degree. When a neighbor is chosen for payoff comparison, the neighbor is likely to be a cooperator.

For networks with a large standardized variance, formula (3) indicates that, on average, the degree of a neighbor will be significantly larger than the degree of a vertex. A cooperator comparing payoff to a cooperator leads to no strategy change. However, the strong defector is in the tenuous position of comparing payoff to a cooperating vertex with, statistically, a significantly higher connectivity. The result will more often be a switch in strategy and a gain for cooperators. This is not to say that strong cooperators are impervious to strategy shifts-it has been shown that most vertices will spend some time employing both game strategies [14] - but on average, strong cooperators are less vulnerable while strong defectors are more so.

To emphasize this dynamic, several networks are included with relatively large heterogeneity given by $\nu_{s t}$, but relatively small maximum vertex degree. These networks are constructed in the same manner as $Z$ series with average degree 4 , with the exception that the degree distribution is then truncated. Included are three networks with maximum degree 50 and $\nu_{s t}=2.18$, and two with maximum degree 60 and $\nu_{s t}$ $=2.558$, which are labeled $Z_{4, s}^{\text {trunc }}$. Despite the lack of large hubs, these networks show cooperation levels consistent with trends formed by the existing nontruncated data.

It has been a widely observed phenomenon that increased average degree usually hinders cooperation. This becomes clear in the denominator of $\nu_{s t}$ in formula (3). If the average degree of the vertices on the network increases without a significant increase in the average degree of the neighbors on the network, then the $\nu_{s t}$ will decrease leading to less cooperation on the network. On the other hand, an increase in $\nu_{s t}$, and therefore cooperation, is not precluded by an increase in average degree, but rather requires an appropriately large increase in the size of an average neighbor.

In order to make the relationship between $\nu_{s t}$ and average cooperation in Fig. 4 precise, we note a connection between $\nu_{s t}$ and epidemic disease spread on networks. The epidemic threshold $\lambda_{c}$ of a disease on a network is the critical disease infectivity below which an epidemic outbreak of the disease on the network is not possible. The lower the epidemic threshold of the network, therefore, the easier it is for a disease to spread, and for a nontrivial fraction of the population to become infected. If the network is uncorrelated, then the epidemic threshold is given by [23]

$$
\lambda_{c}=\frac{\langle k\rangle}{\left\langle k^{2}\right\rangle} .
$$

It follows from Eq. (2) that $\lambda_{c}=\frac{1}{T^{\prime}(1)}$. Intuitively, the data shows that cooperation also spreads more successfully on networks with a lower epidemic threshold.

Figure 5 is a plot showing average weighted cooperation as a function of the epidemic threshold of the network $\lambda_{c}$. The relationship is clear: average cooperation increases linearly by average degree as epidemic threshold decreases.

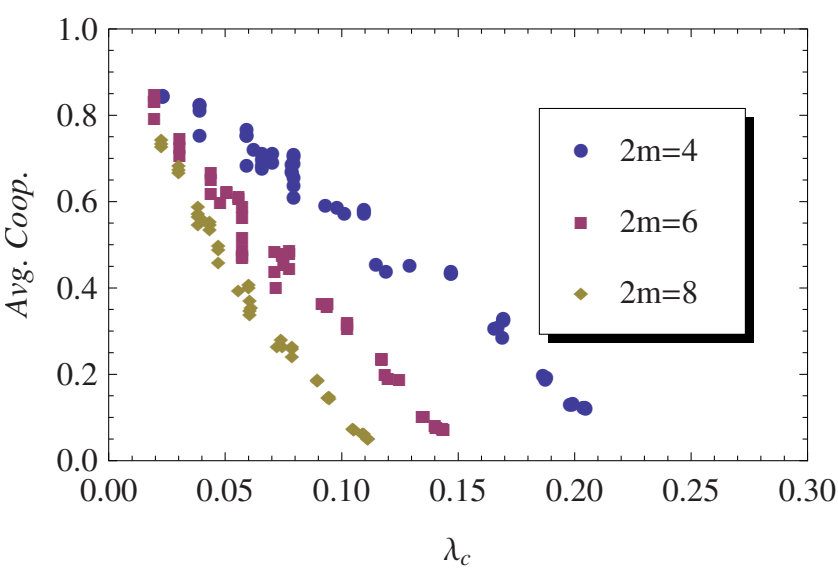

FIG. 5. (Color online) Average cooperation as a function of the network's epidemic threshold $\lambda_{c}$.

The fact that the trends in average cooperation as a function of $\lambda_{c}$ are degree dependent is not at all surprising in light of Eq. (5). The epidemic threshold depends only on the average degree of a neighbor, $T^{\prime}(1)$, and the size of an average vertex is irrelevant. In the evolutionary PD, however, the average size of the vertex is relevant as it is an indicator of the strength of the vertex in relation to the neighbor, and hence, of the susceptibility of the vertex to become "infected" by cooperation. For example, if a network $\mathcal{N}$ has average neighbor $T^{\prime}(1)=20$, then the epidemic threshold is $\lambda_{c}=0.05$ regardless of average vertex size. However, in a network with average degree $G^{\prime}(1)=4$, a neighbor is considerably stronger than in a network with average degree $G^{\prime}(1)=8$. In the former case, the average neighbor is five times the size of the average vertex whereas in the latter, an average neighbor is only 2.5 times the size of an average vertex. Therefore multiplying the epidemic threshold $\lambda_{c}$ by the average network degree gives a universal parameter that can be compared across networks of varying average degree.

It follows easily from Eqs. (3) and (5) that

$$
\langle k\rangle \lambda_{c}=\frac{G^{\prime}(1)}{T^{\prime}(1)}=\frac{1}{1+\nu_{s t}} .
$$

In Fig. 6, average cooperation is plotted as a function of $\langle k\rangle \lambda_{c}=\frac{1}{1+\nu_{s t}}$. The result is a striking, but now not unexpected, linear relationship. Average network cooperation depends linearly on the ratio of average vertex degree to average neighbor degree, a quantity that is simply the network's epidemic threshold adjusted by the network's average degree. Moreover, both quantities are simple functional variants of standardized variance, $\nu_{s t}$. The linear correlation between $\bar{c}_{\mathcal{N}}$ and $\langle k\rangle_{\mathcal{N}} \lambda_{c, \mathcal{N}}$ is particularly strong, with correlation coefficient $r=0.981\left(r^{2}=0.962\right)$. While cooperation in an evolutionary PD on a network and susceptibility of the network to epidemic outbreaks is an intuitive connection [30], this is an instance of such a relationship in a simple, quantifiable way.

It is also interesting to consider the possible reasons for fluctuations in Fig. 6. The first is finite size effects. All networks considered here have maximum vertex size capped at 600 to minimize such effects on the dynamics. However, in a 


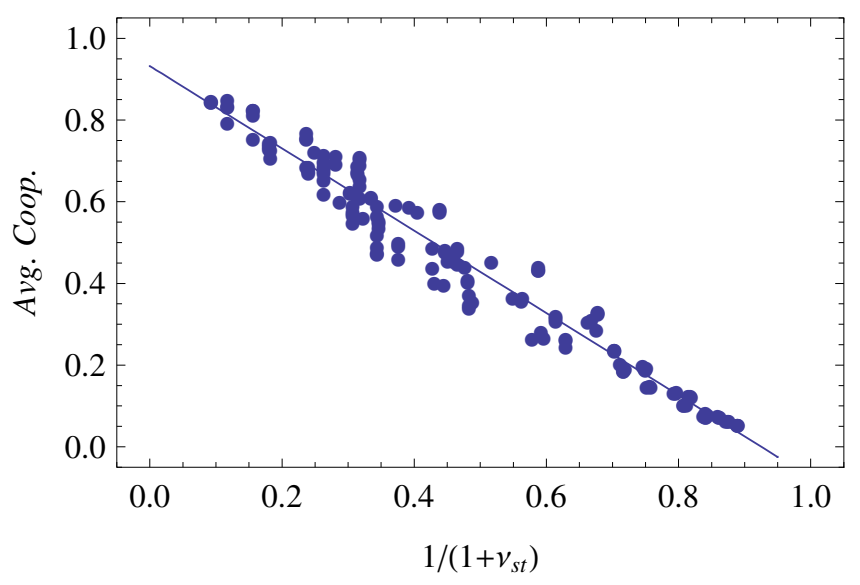

FIG. 6. (Color online) Average cooperation as a function of $\langle k\rangle \lambda_{c}=\frac{1}{1+\nu_{s t}}$. In addition, the figure shows the linear regression line $y=(-1.0074 x+0.9322)$

network of $10^{4}$ vertices, a vertex of degree 600 reaches nearly $6 \%$ of the network's total vertices. If a network has several very large vertices, likely interconnected, unforseen impacts on the dynamics are certainly possible. Relaxing the cap on maximum vertex size for networks with wide tails (and many large vertices) does seem to have a negative impact on cooperation.

The second explanation for deviations from the regression line, especially in graphs with wide tails, is the possible presence of small scale correlations between the degrees of vertices at either end of an edge. Despite the use of the configuration model, it is possible for local contact information to result in correlations that can impact the dynamics.

\section{CONCLUSIONS}

We have used standardized variance to measure heterogeneity in networks that are maximally random with a fixed degree distribution. By studying an evolutionary PD on these networks for a wide class of degree distributions with varying levels of heterogeneity, we have shown that $\nu_{s t}$ is a powerful indicator of the success of global cooperation in the evolutionary PD. This result is particularly striking given the simplicity of $\nu_{s t}$ and the complexity of the dynamics being studied. The ease of calculation of $\nu_{s t}$, and the ability to compare $\nu_{s t}$ across networks with different average degrees and distributions provides a strong case for $\nu_{s t}$ as a reasonable quantification of the network heterogeneity concept for random networks. Moreover, the expression for $\nu_{s t}$ coming from the generating functions associated to the network, as a strength of neighbor relationship between average degrees of vertices and neighbors, gives valuable insight into the mechanisms that contribute to enhanced cooperation. This interpretation and the methods used in this paper can be generalized to yield analogous concepts for networks where degree-degree correlations are present. That similar results might hold for evolutionary games on these networks is a promising direction for future work.

Standardized variation provides a possible pathway for understanding the connection between cooperation phenomena and epidemic spread of diseases, two fields that are intuitively related and share as foundational the study of complex networks. The relationship between $\nu_{s t}$ and cooperation leads directly to a connection between network cooperation and the epidemic threshold of the network. That connection, pictured in Fig. 6, is particularly interesting and indicates a deep relationship between the dynamics of the two systems that can be further explored.

\section{ACKNOWLEDGMENTS}

The authors thank Peter Pacheco for useful discussions and programming expertise.
[1] R. Axelrod, J. Conflict Resolut. 24, 3 (1980).

[2] R. Axelrod, The Evolution of Cooperation (Basic Books, New York, 1984).

[3] H. Gintis, Game Theory Evolving (Princeton University, Princeton, NJ, 2000).

[4] J. Maynard Smith, Evolution and the Theory of Games (Cambridge University Press, Cambridge, England, 1982).

[5] Genetic and Cultural Evolution of Cooperation, edited by Peter Hammerstein (MIT, Cambridge, MA, 2003).

[6] P. E. Turner and L. Chao, Nature (London) 398, 441 (1999).

[7] J. Hofbauer and K. Sigmund, Evolutionary Games and Population Dynamics (Cambridge University Press, Cambridge, England, 1998).

[8] M. A. Nowak and R. M. May, Nature (London) 359, 826 (1992).

[9] G. Szabó and G. Fáth, Phys. Rep. 446, 97 (2007).

[10] F. C. Santos and J. M. Pacheco, Phys. Rev. Lett. 95, 098104 (2005).

[11] F. C. Santos and J. M. Pacheco, J. Evol. Biol. 19, 726 (2006).
[12] F. C. Santos, J. M. Pacheco, and T. Lennaerts, Proc. Natl. Acad. Sci. U.S.A. 103, 3490 (2006).

[13] F. C. Santos, J. F. Rodrigues, and J. M. Pacheco, Proc. R. Soc. London, Ser. B 273, 51 (2005).

[14] J. Gómez-Gardeñes, M. Campillo, L. M. Floría, and Y. Moreno, Phys. Rev. Lett. 98, 108103 (2007).

[15] J. Poncela, J. Gómez-Gardeñes, L. M. Floría, and Y. Moreno, New J. Phys. 9, 184 (2007).

[16] A. Pusch, S. Weber, and M. Porto, Phys. Rev. E 77, 036120 (2008).

[17] G. Abramson and M. Kuperman, Phys. Rev. E 63, 030901(R) (2001).

[18] R. Albert and A.-L. Barabási, Science 589, 209 (1999).

[19] R. Albert and A.-L. Barabási, Rev. Mod. Phys. 74, 47 (2002).

[20] S. N. Dorogotsev and J. F. F. Mendes, Evolution of Networks: From Biological Nets to the Internet and WWW (Oxford University, Oxford, 2003).

[21] M. E. J. Newman, S. H. Strogatz, and D. J. Watts, Phys. Rev. E 64, 026118 (2001). 
[22] M. Barthélemy, A. Barrat, R. Pastor-Satorras, and A. Vespignani, J. Theor. Biol. 235, 275 (2005).

[23] Y. Moreno, R. Pastor-Satorras, and A. Vespignani, Eur. Phys. J. B 26, 521 (2002).

[24] J. Gómez-Gardeñes and Y. Moreno, Phys. Rev. E 73, 056124 (2006).

[25] P. Erdös and A. Rényi, Publ. Math. (Debrecen) 6, 290 (1959).

[26] P. Erdös and A. Rényi, Publ. Math. Inst. Hung. Acad. Sci. 5, 17 (1960).

[27] P. Erdös and A. Rényi, Publ. Math. Inst. Hung. Acad. Sci. 12, 261 (1961).
[28] M. Molloy and B. Reed, Random Struct. Algorithms 6, 161 (1995).

[29] When the tail of the power law describing the degree distribution has an exponent close to 3 the statistics show the most noise, presumably due to the added impact of local network structure, and therefore, initial configurations. In some of these cases, additional networks (not more than three) have been included to get a more accurate picture of the dynamics.

[30] F. C. Santos, J. F. Rodrigues, and J. M. Pacheco, Phys. Rev. E 72, 056128 (2005). 